\section{§30. Real-Time Plasma Control System for LHD}

Nishimura, K., Takami, S., Takahashi, C., Chikaraishi, H., Yamazaki, K.

For the steady-state operation in LHD, real-time plasma control (RTPC) is one of the most essential techniques. Controlled plasma parameters by the RTPC system are plasma density, ion and electron temperature, plasma current, cross-sectional shape, position of plasma boundary and magnetic axis, and wall loading. Externally controllable parameters are a gas puffing rate, the number and the repetition time of injecting pellets, heating power of ECH, NBI and ICRF, and a combination of coil currents (one pair of helical coil currents and three pairs of poloidal coil currents). Each device forms an individual system called sub-system. They are controlled and operated by their own control computers. The role of the RTPC computer is analyzing data (plasma parameters) sent from the data acquisition computers and sending the control parameters, such as the fueling rate, coil currents, heating powers and so on, to the computers of the sub-systems through the local network.

Conceptual diagram of the RTPC system is shown in Fig.1. To get good control performance, this RTPC system is required a good time response, flexibility and easiness of extension of the input and output parameters.

As the first step of the RTPC system, we designed the real-time plasma shape control system. This system is linked to the poloidal field coil control computer (VME) through a shared memory as shown in Fig. 2. Plasma parameters of toroidal field $\mathrm{B}_{0}$, plasma current $\mathrm{I}_{\mathrm{p}}$, plasma position $\Delta$ and an ellipticity $\kappa$ of a plasma cross-section are sent to the Plasma Shape control computer directly or through the LAN. Data set of the coil currents are calculated and sent to the shared memory every $20 \mathrm{msec}$. The PF coil control computer calculates current changing rates for all coil sets using these data and sends them to the HF coil control computer.

All interlock systems and the security systems for the power supplies are kept as beforre.

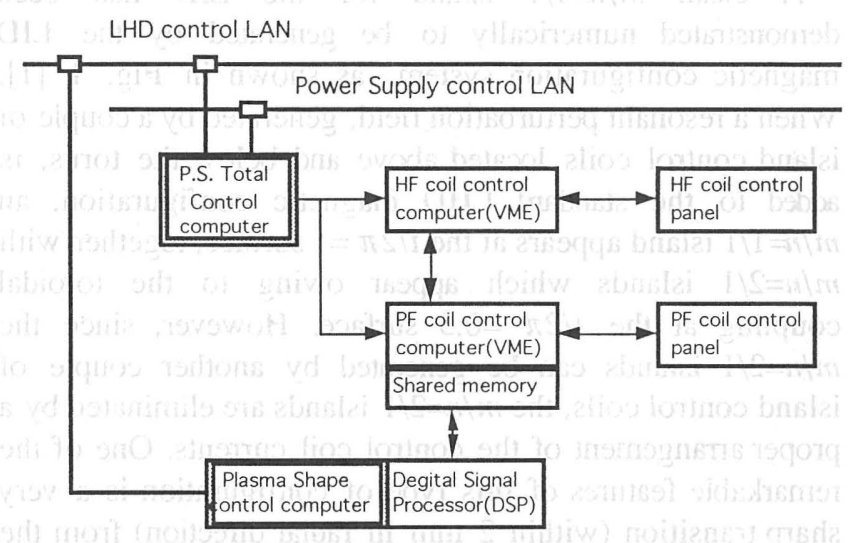

Fig. 2. Conceptual diagrm of the Power Supply and the Plasma Shape Control systems.

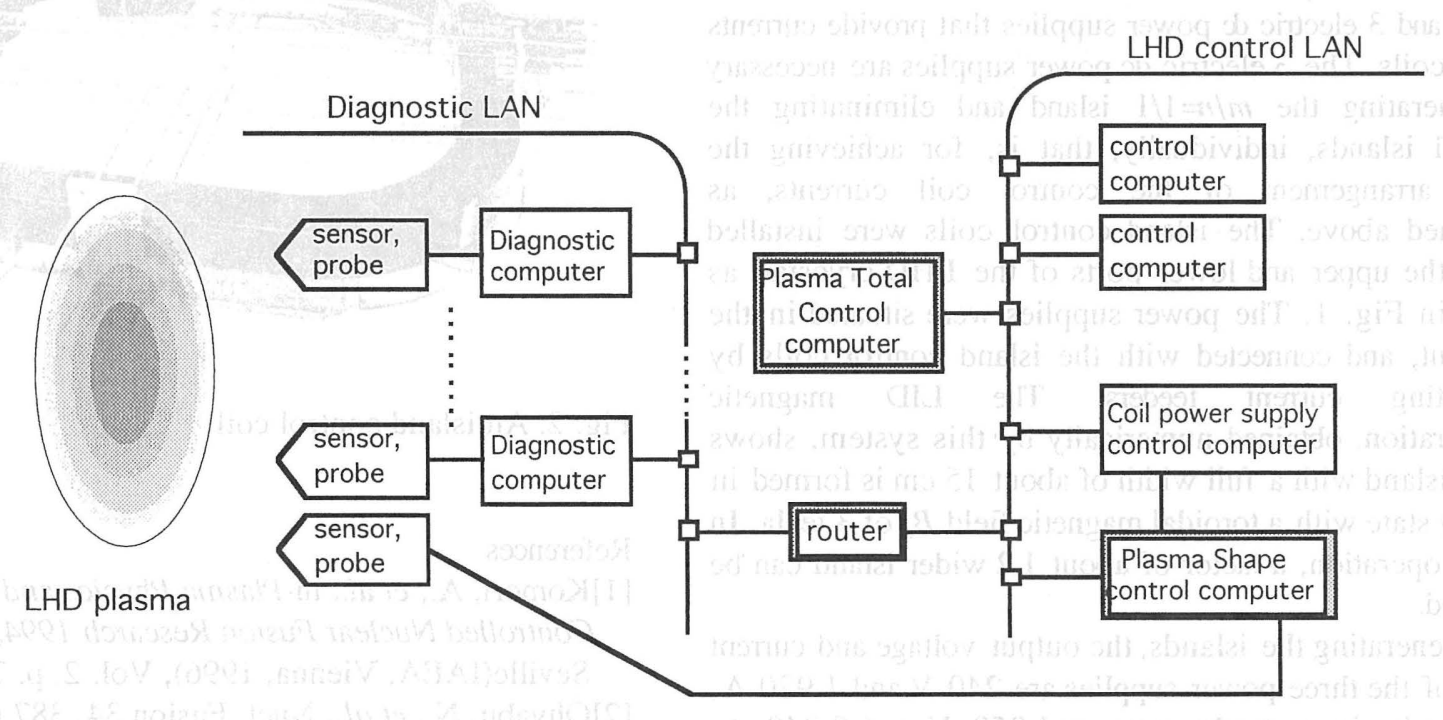

Fig.1. Conceptual diagrm of the Real-Time Plasma Control system. 